\title{
Investigation of Variation in Phenotype and DNA Content between Single-conidium Isolates of Single Penicillium Strains
}

\author{
By P. D. BRIDGE, ${ }^{1 *}$ L. HUDSON,${ }^{2}$ Z. KOZAKIEWICZ, ${ }^{3}$ A. H. S. ONIONS ${ }^{1}$ \\ AND R. R. M. PATERSON ${ }^{1}$ \\ ${ }^{\prime} C A B$ International Mycological Institute, Ferry Lane, Kew, Surrey TW9 3AF, UK \\ 2 Department of Immunology, St George's Hospital Medical School, Cranmer Terrace, \\ London SWI7 ORE, UK \\ ${ }^{3}$ Ministry of Agriculture, Fisheries and Food, Slough Laboratory, London Road, Slough, \\ Berkshire SL3 7HJ, UK
}

(Received 30 October 1986)

\begin{abstract}
Variation in phenotypic properties was examined in three strains of closely related fasciculate species of Penicillium using 114 morphological, physiological, and biochemical characters. Thirty-six of these characters showed variation within single-conidium isolates of the same strain. Conidial sizes and nuclear DNA contents were compared using flow microfluorimetry; these results suggested that significant differences in conidial DNA content are associated with phenotypic variation. The taxonomic significance of the results is discussed.
\end{abstract}

\section{INTRODUCTION}

Variability in properties and the spontaneous occurrence of variant lines within single strains has been reported in many genera of filamentous fungi, including Penicillium (e.g. Hooker, 1957; Scharen \& Krupinsky, 1970; Bridge et al., 1986c). This variability may have considerable significance both in the selection and maintenance of strains of industrial importance and in the construction and use of taxonomic schemes. The taxonomic situation within Penicillium has been reviewed several times in recent years, with a variety of properties being employed (see Onions et al., 1984; Samson \& Gams, 1984). The majority of these studies have reported significant variability within species, with some strains appearing to intergrade between 'species'.

We have shown that significant variation in both morphological and biochemical features may give rise to distinct lines within the ex-type strain of $P$. viridicatum (Bridge et al., 1986c). These studies showed differences in morphology and secondary metabolite production, and flow-cytometric results suggested that there may be a correlation between variants and ploidy levels within that strain (Bridge et al., 1986 b). The effects of ploidy levels on particular properties have been studied for a number of fungal genera, and heteroploidy has been proposed as a mechanism of variation (Pathak \& Elander, 1971; Kafer \& Upshall, 1973; Tolmsoff, 1983). However, it is not clear how widespread this phenomenon is or to what extent this process may operate in nature.

In order to construct reliable taxonomic schemes, it is important that the occurrence and degree of variability is investigated. Variation must be considered not only between strains of individual taxa but also within single strains. This paper describes the variations in phenotype and DNA content in five single-conidium isolates of each of three closely related penicillia.

Abbreviations: FALS, forward angle light scatter; LIGFL, log integral green fluorescent light; SEM, scanning electron microscopy. 


\section{METHODS}

Isolates studied. Five separate single-conidium cultures were obtained from each of Penicillium granulatum (IMI 296059), designated A-E, and P. martensii (IMI 297891), designated F-J. Four separate single-conidium cultures were obtained from $P$. crustosum (IMI 291543) and one of these was duplicated and maintained separately; these were designated $\mathrm{K}-\mathrm{O}$, with $\mathrm{N}$ being a duplicate of $\mathrm{M}$. Cultures were maintained on slopes of Czapek agar (Cz; Smith \& Onions, 1983).

Strain characterization. The 15 lines from the single-conidium cultures were characterized using the features described by Bridge et al. (1986a) (listed in Tables 1 and 2). Tests were done in duplicate (except for TLC of strains $\mathrm{A}, \mathrm{L}$ and $\mathrm{N}$, which was done in triplicate) at the same time using media and reagents from the same batches.

Morphological properties. Morphological features, including cultural characteristics, were observed for all isolates after $7 \mathrm{~d}$ incubation in the dark at $25^{\circ} \mathrm{C}$ on $\mathrm{Cz}$ agar plates. These features were scored as 53 individual characters using the scheme given by Bridge et al. (1986a) and the terminology of Minter et al. (1986).

Ultrastructural properties. Ultrastructural features of conidia were observed using an S250 Cambridge Scientific Instruments scanning electron microscope. Samples were prepared by rubbing an aluminium stub (Agar Aids) across a $30 \mathrm{~d}$ colony. This was gold-coated (Emscope sputter-coater) and examined at an accelerating voltage of $20 \mathrm{kV}$.

Physiological and biochemical properties. Enzyme production, growth under different conditions, and growth and final pH on carbon and nitrogen sources were tested using the methods of Bridge (1985) and Bridge et al. (1986a). The production of yellow colonies was tested on $\mathrm{Cz}$ agar with the sodium nitrate replaced by ammonium oxalate $\left(\mathrm{CzNH}_{4}\right)$ using the method of Bridge et al. (1986c). This medium apparently stimulates the production of yellow pigment in some penicillia. Growth on media of reduced water activity was tested by measuring colony diameters on G25N agar (Pitt, 1980). Growth and final pH on creatine was tested using both liquid media (Bridge, 1985) and creatine/sucrose agar (Frisvad, 1981). Similarly, growth with sodium nitrite as sole nitrogen source was tested in liquid media at pH 4.5 (Bridge, 1985) and on nitrite/sucrose agar at pH 7 (Frisvad, 1981). Inhibition of growth by copper sulphate, crystal violet and malachite green was measured as the diameter of inhibition zones in a $\mathrm{Cz}$ plate seeded with fungal spores ( $1 \mathrm{ml}$ of a $2 \times 10^{6}$ spore suspension in $10 \mathrm{ml} \mathrm{Cz}$ agar). Separate filter paper discs each impregnated with inhibitor $(5,0.05$ and $0.04 \%, w / v$, respectively) were aseptically placed on each plate after seeding. Diameters were measured after $7 \mathrm{~d}$ incubation at $25^{\circ} \mathrm{C}$.

Secondary metabolite production. This was tested using the agar plugs and TLC techniques employed by Paterson (1986).

Stability. The stability of the single-conidium lines was estimated using line A of P. granulatum. This culture produced single-conidium lines that differed in gross colony morphology, so that differences could be easily visualized. Aged ( $21 \mathrm{~d}$ ) cultures of line A were used to prepare conidial suspensions in aqueous $0 \cdot 2 \%(\mathrm{v} / \mathrm{v}) \mathrm{Tween}$ $80\left(10^{6}\right.$ conidia $\left.\mathrm{ml}^{-1}\right)$. Samples $(0.1 \mathrm{ml})$ from each serial 1 -fold dilution of this suspension were spread onto $\mathrm{Cz}$ plates and incubated at $25^{\circ} \mathrm{C}$ for $7 \mathrm{~d}$.

Conidial nuclear DNA content. This was estimated by continuous flow microfluorimetry with conidial suspensions. Conidia were fixed in Carnoy's fluid and stained by the Feulgen/acriflavine technique described by Bridge et al. ( $1986 \mathrm{~b}$ ). Conidial sizes and DNA contents were then estimated for 10000 conidia in each sample with an EPICS V flow cytometer (Coulter Instruments) using a laser setting of $300 \mathrm{~mW}$ at $488 \mathrm{~nm}$. Conidial sizes were compared by measuring forward angle light scatter (FALS) alone, $90^{\circ}$ light scatter giving no added information. DNA contents were compared using emitted fluorescence and measuring log integral green fluorescent light (LIGFL) detected using $515 \mathrm{~nm}$ long pass interference and absorbance filters. DNA measurements were made using 'gated' populations, where only particles within the size range of the conidia were recorded (Bridge et al., $1986 b$ ).

Numerical methods. The dendrogram (Fig. 1) was produced using the NUMIPAC computer package (Bridge \& May, 1984) adapted to run on an Amstrad PCW8512 microcomputer. Similarities were expressed using the taxonomic distance coefficient $\left(D_{t}\right)$ and clustered using UPGMA average linkage (Sneath \& Sokal, 1973).

\section{RESULTS}

The results from the phenotypic characterization that showed significant differences between isolates of the same strain are given in Table 1 . All measurements are given as means in $\mathrm{mm}$; all of these showed confidence limits of within $\pm 2.5 \mathrm{~mm}$. Characters that did not vary significantly between isolates of the same strain are given in Table 2 . A dendrogram was constructed from the results given in Table 1 and those characters in Table 2 that gave differential results for the three parental isolates. This dendrogram, based on 44 characters, is shown in Fig. 1. The different lines of $P$. martensii $(\mathrm{F}-\mathrm{J})$ and $P$. crustosum $(\mathrm{K}-\mathrm{O})$ were recovered in two distinct clusters corresponding with those parental strains. The duplicated lines $(\mathrm{M}$ and $\mathrm{N})$ appeared adjacent to 
Table 1. Phenotypic characters showing significant variation between single-conidium lines

\begin{tabular}{|c|c|c|c|c|c|c|c|c|c|c|c|c|c|c|c|}
\hline \multirow[b]{2}{*}{ Character } & \multicolumn{5}{|c|}{ P. granulatum } & \multicolumn{5}{|c|}{ P. martensii } & \multicolumn{5}{|c|}{ P. crustosum } \\
\hline & A & B & $\mathrm{C}$ & $\mathrm{D}$ & $\mathrm{E}^{\prime}$ & $\mathbf{F}$ & G & $\mathrm{H}$ & I & J & $\mathrm{K}$ & $\mathrm{L}$ & $\mathbf{M}$ & $\mathbf{N}$ & $\mathrm{O}$ \\
\hline \multicolumn{16}{|l|}{ Physiology } \\
\hline Growth at $4^{\circ} \mathrm{C}$ & + & + & + & + & + & + & $\mathbf{w}$ & + & + & - & + & + & + & + & $\mathbf{w}$ \\
\hline Growth at pH 2 & - & $\mathbf{w}$ & $\mathbf{w}$ & - & + & - & - & - & - & - & $\mathbf{w}$ & $\mathbf{w}$ & - & - & - \\
\hline \multicolumn{16}{|l|}{ Colony diameter on } \\
\hline $\mathrm{G} 25 \mathrm{~N}$ agar $(\mathrm{mm})$ & 10 & 11 & 23 & 24 & 26 & 24 & 23 & 25 & 23 & 24 & 23 & 26 & 23 & 24 & 20 \\
\hline \multicolumn{16}{|l|}{ Inhibition by copper sulphate } \\
\hline $\operatorname{discs}(\mathrm{mm})$ & 23 & 27 & 26 & 18 & 24 & 30 & 18 & 26 & 21 & 22 & 42 & 10 & 12 & 16 & 17 \\
\hline \multicolumn{16}{|l|}{ Inhibition by crystal violet } \\
\hline $\operatorname{discs}(\mathrm{mm})$ & 15 & 15 & 9 & 10 & 9 & 9 & 8 & 5 & 8 & 5 & 12 & 11 & 12 & 11 & 10 \\
\hline \multicolumn{16}{|l|}{ Inhibition by malachite green } \\
\hline $\begin{array}{l}\text { discs }(\mathrm{mm}) \\
\text { Gelatin liquefaction }\end{array}$ & $\begin{array}{l}11 \\
+\end{array}$ & $\begin{array}{l}12 \\
+\end{array}$ & $\begin{array}{l}12 \\
+\end{array}$ & $\begin{array}{l}10 \\
+\end{array}$ & $\begin{array}{r}5 \\
+\end{array}$ & $\begin{array}{r}9 \\
+\end{array}$ & $\begin{array}{r}7 \\
+\end{array}$ & $\begin{array}{r}9 \\
+\end{array}$ & $\begin{array}{r}6 \\
+\end{array}$ & $\begin{array}{l}11 \\
+\end{array}$ & $\begin{array}{r}8 \\
+\end{array}$ & 7 & $\begin{array}{c}12 \\
+\end{array}$ & 7 & $\begin{array}{r}9 \\
+\end{array}$ \\
\hline Pectate liquefaction ( $\mathrm{pH} 8)$ & - & - & - & - & - & - & - & - & - & $\begin{array}{l}\top \\
-\end{array}$ & + & + & $\begin{array}{l}\top \\
-\end{array}$ & - & + \\
\hline RNA hydrolysis & - & - & - & - & + & + & + & + & + & + & - & - & + & + & - \\
\hline Selenite reduction & - & - & + & + & - & + & + & + & + & + & - & + & - & - & - \\
\hline \multicolumn{16}{|l|}{ Carbon source } \\
\hline Base from citric acid & - & - & + & $\mathbf{w}$ & + & + & $\mathbf{w}$ & + & + & + & + & + & + & + & $\mathbf{w}$ \\
\hline \multicolumn{16}{|l|}{ Nitrogen source } \\
\hline \multicolumn{12}{|l|}{ Growth on sodium nitrite } & & & $\mathbf{w}$ & - \\
\hline \multicolumn{16}{|l|}{ Growth on sodium nitrite } \\
\hline (pH 7, agar medium) & + & + & - & - & - & - & - & - & - & - & - & - & - & - & - \\
\hline Base from creatine agar & - & - & + & + & $\mathbf{w}$ & + & + & + & + & + & + & + & + & + & + \\
\hline Base from glycine & + & + & + & $w$ & $w$ & + & + & + & - & - & - & - & - & - & - \\
\hline Base from ammonium oxalate & w & - & $\mathbf{w}$ & $\mathbf{w}$ & + & + & + & $\mathbf{w}$ & $\mathbf{w}$ & $\mathbf{w}$ & + & + & + & + & w \\
\hline \multicolumn{16}{|l|}{ Morphology } \\
\hline Colony diameter (mm) & 18 & 16 & 17 & 17 & 35 & 27 & 28 & 28 & 27 & 27 & 26 & 27 & 27 & 26 & 27 \\
\hline Colony umbonate & + & + & + & - & + & + & + & + & + & + & + & + & + & + & + \\
\hline Colony wrinkled & - & - & - & - & - & $\mathbf{w}$ & $\mathbf{w}$ & $\mathbf{w}$ & $\mathbf{w}$ & $\mathbf{w}$ & + & $\mathbf{w}$ & $\mathbf{w}$ & $\mathbf{w}$ & $\mathbf{w}$ \\
\hline Conidia blue-green & + & + & + & + & + & + & + & + & + & + & + & - & + & + & + \\
\hline Conidia green & - & - & - & - & - & + & - & - & - & - & - & - & - & + & - \\
\hline Conidia dark green & - & - & - & - & - & - & - & - & - & - & - & + & + & - & - \\
\hline Straw-coloured exudate & + & + & + & + & - & - & - & + & - & + & + & + & - & + & - \\
\hline Yellow exudate & + & + & + & + & - & - & - & - & - & - & - & - & - & - & - \\
\hline Yellow pigment & + & + & + & + & - & - & - & - & - & - & - & - & - & - & - \\
\hline Reverse white & - & - & - & - & + & + & + & + & + & + & + & + & + & + & + \\
\hline Reverse brown & + & + & + & + & - & - & - & - & - & - & - & - & - & - & - \\
\hline Reverse pink & - & - & + & - & - & - & - & - & - & - & + & + & + & + & + \\
\hline Black/grey areas in reverse & - & - & + & - & - & + & + & + & + & + & - & - & - & - & - \\
\hline Supporting hyphae: swollen & - & - & - & - & - & - & - & - & - & - & - & - & + & - & - \\
\hline \multicolumn{16}{|l|}{ Electron microscopy } \\
\hline \multicolumn{8}{|l|}{ Conidial shape and } & & 3 & 3 & 2 & 2 & 1 & 1 & 2 \\
\hline \multicolumn{16}{|l|}{ Metabolites detected by TLC } \\
\hline Brefeldin A & - & - & - & - & - & - & - & - & - & - & + & + & - & - & - \\
\hline 11-Deacetoxywortmannin & - & + & - & - & - & - & - & - & - & - & - & - & - & - & - \\
\hline Roquefortine B & - & - & - & - & + & - & - & - & - & - & + & + & + & + & + \\
\hline Roquefortine C & - & - & - & - & + & - & - & - & - & + & - & - & - & - & - \\
\hline
\end{tabular}

- Examples of the three types of conidia are shown in Fig. 2(a-c).

each other as a distinct pair. Lines of $P$. granulatum (A-E) did not form a distinct cluster, appearing as two pairs $(\mathrm{A}+\mathrm{B}$ and $\mathrm{C}+\mathrm{D})$, with line $\mathrm{E}$ being loosely linked to the $P$. martensii$P$. crustosum clusters.

Examples of the variation in conidial shape and ornamentation as seen by electron microscopy are given in Fig. $2(a-c)$. Conidial sizes varied both between and within singleconidium isolates. In general, conidia of topography type 1 were small (length approx. $2-2.5 \mu \mathrm{m}$ ) 
Table 2. Characters that showed no variation between single-conidium lines of the same strain

Growth at $30^{\circ} \mathrm{C}^{b}$

Growth at $40^{\circ} \mathrm{Cc}^{\mathrm{c}}$

Growth at $\mathrm{pH} 12^{\mathrm{c}}$

Growth on sodium azide $\left(0.1 \mathrm{~g}^{-1}\right)^{b}$

Pectate liquefaction $\mathrm{pH} 6^{b}$

Aesculin hydrolysis ${ }^{b}$

Cellulase ${ }^{a}$

Starch hydrolysis ${ }^{b}$

Tellurite reduction $\left(0.032 \mathrm{~g} \mathrm{l}^{-1}\right)^{b}$

Growth on ammonium tartrate

Growth on ammonium oxalate ${ }^{c}$

Growth on tannic acid ${ }^{b}$

Growth on lactic acid

Growth on creatine (liquid $\mathrm{pH} 4 \cdot 5)^{b}$

Growth on urea ${ }^{b}$

Growth on glycine ${ }^{b}$

Colony depth

Colony flat/raised

Mycelium white ${ }^{b}$

Colony velvet ${ }^{c}$

Colony funiculose ${ }^{c}$

Colony margin submerged ${ }^{b}$

Conidia grey/green ${ }^{b}$

Conidia white ${ }^{c}$

Pink-red exudate

Brown pigment ${ }^{c}$

Red pigment ${ }^{c}$

Reverse greenc

Reverse yellow

Conidial head size

Supporting hyphae rough ${ }^{b}$

One level of branches: asymmetric

Two or more levels of branches ${ }^{b}$

First-level branches: divergent ${ }^{c}$

First-level branches: rough ${ }^{a}$

First-level branches: length ${ }^{a}$

Conidiogenous cells: numbers ${ }^{a}$
Physiology

Growth at $37^{\circ} \mathrm{C}^{\mathrm{c}}$

Growth at $\mathrm{pH} 10^{b}$

Growth on pentachlorophenol $\left(0.008 \mathrm{~g}^{-1}\right)^{b}$

Growth on sodium selenite $\left(0.032 \mathrm{~g} \mathrm{l}^{-1}\right)^{b}$

DNA hydrolysis ${ }^{c}$

Casein hydrolysis ${ }^{b}$

Tween 80 hydrolysis $^{b}$

Tetrazolium reduction $\left(0.064 \mathrm{~g} \mathrm{l}^{-1}\right)^{b}$

Yellow colonies on $\mathrm{C}_{2} \mathrm{NH}_{4}$ agar

Carbon sources

Base from ammonium tartrate $e^{b}$

Base from ammonium oxalate ${ }^{c}$

Growth on citric acid ${ }^{b}$

Base from lactic acid ${ }^{b}$

Nitrogen sources

Growth on creatine (agar pH 8$)^{b}$

Base from urea ${ }^{c}$

Growth on ammonium oxalate

Morphology

Colony density

Colony convex/domed

Mycelium yellow ${ }^{c}$

Colony floccose ${ }^{c}$

Colony fasciculate ${ }^{a}$

Conidia yellow/greenc

Conidia fawn ${ }^{c}$

Colourless exudate ${ }^{b}$

Brown exudate ${ }^{c}$

Pink pigment ${ }^{c}$

Reverse buff ${ }^{b}$

Reverse redc

Conidial head arrangement

Supporting hyphae: length

Branches absent ${ }^{b}$

One level of branches: symmetric ${ }^{c}$

First-level branches: numbers ${ }^{a}$

First-level branches: appressed ${ }^{b}$

First-level branches: base cylindrical ${ }^{b}$

Conidiogenous cells: length ${ }^{a}$

Conidiogenous cells: ampulliform ${ }^{b}$

Ergosterol

Metabolites detected by TLC*

Unidentified metabolite U (Paterson, 1986)

Gentisic acida

${ }^{a}$ Characters differentiating parental cultures.

${ }^{b}$ Characters positive for all cultures.

c Characters negative for all cultures.

* A further 83 named or characterized secondary metabolites visualized by the methods used were not found in any of these isolates.

and conidia of topography type 3 were large (length approx. 4-5 $\mu \mathrm{m}$ ), with topography type 2 conidia being of intermediate size (length 3-4 $\mu \mathrm{m}$ ). However, these classes were not absolute, since limited numbers of large conidia were apparent in the type 1 populations and limited numbers of small conidia in the type 3 populations (Fig. $2 d$ ).

The assessment of stability for the single-conidium line A showed results ranging from 1 to $10 \%$ for the frequency of occurrence of yellow-pigment-deficient colonies (Fig. 3). Repeated single-conidium isolation from the parental $P$. granulatum culture gave results broadly in agreement with those reported above. This process did however produce a further set of six 


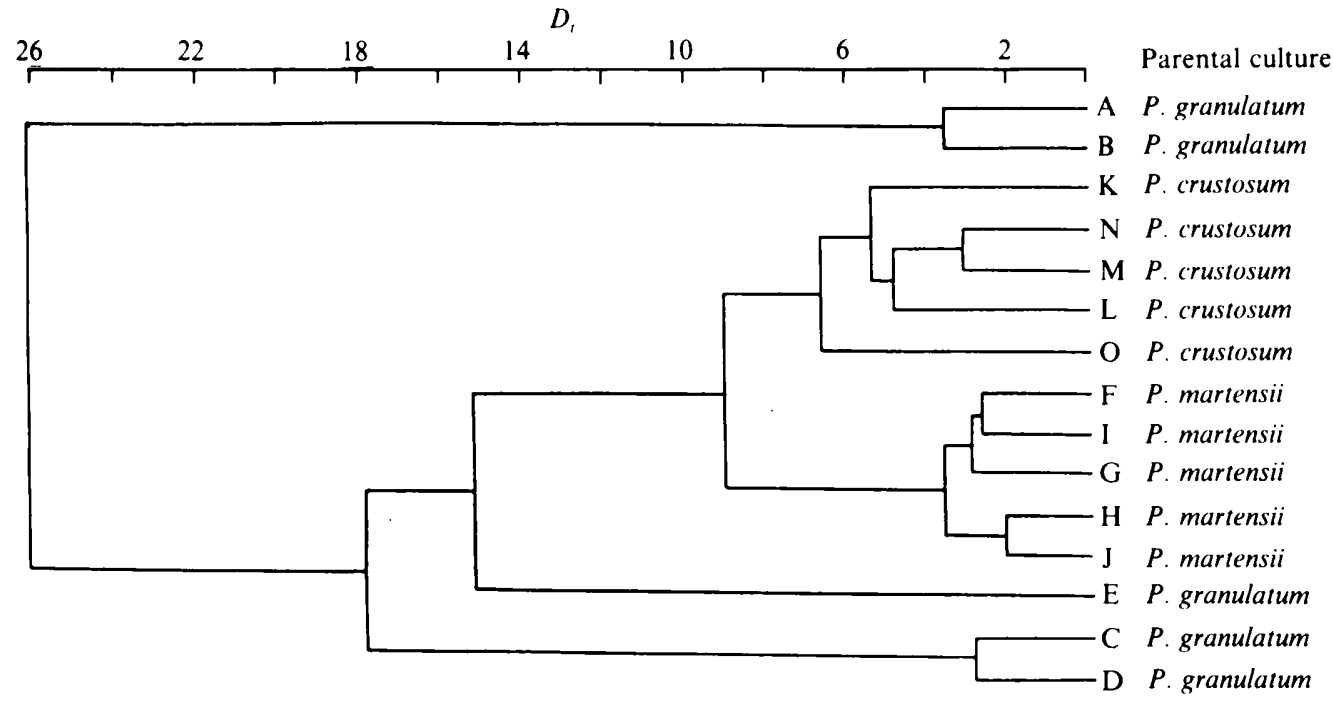

Fig. 1. UPGMA dendrogram constructed from the 44 variable characters.
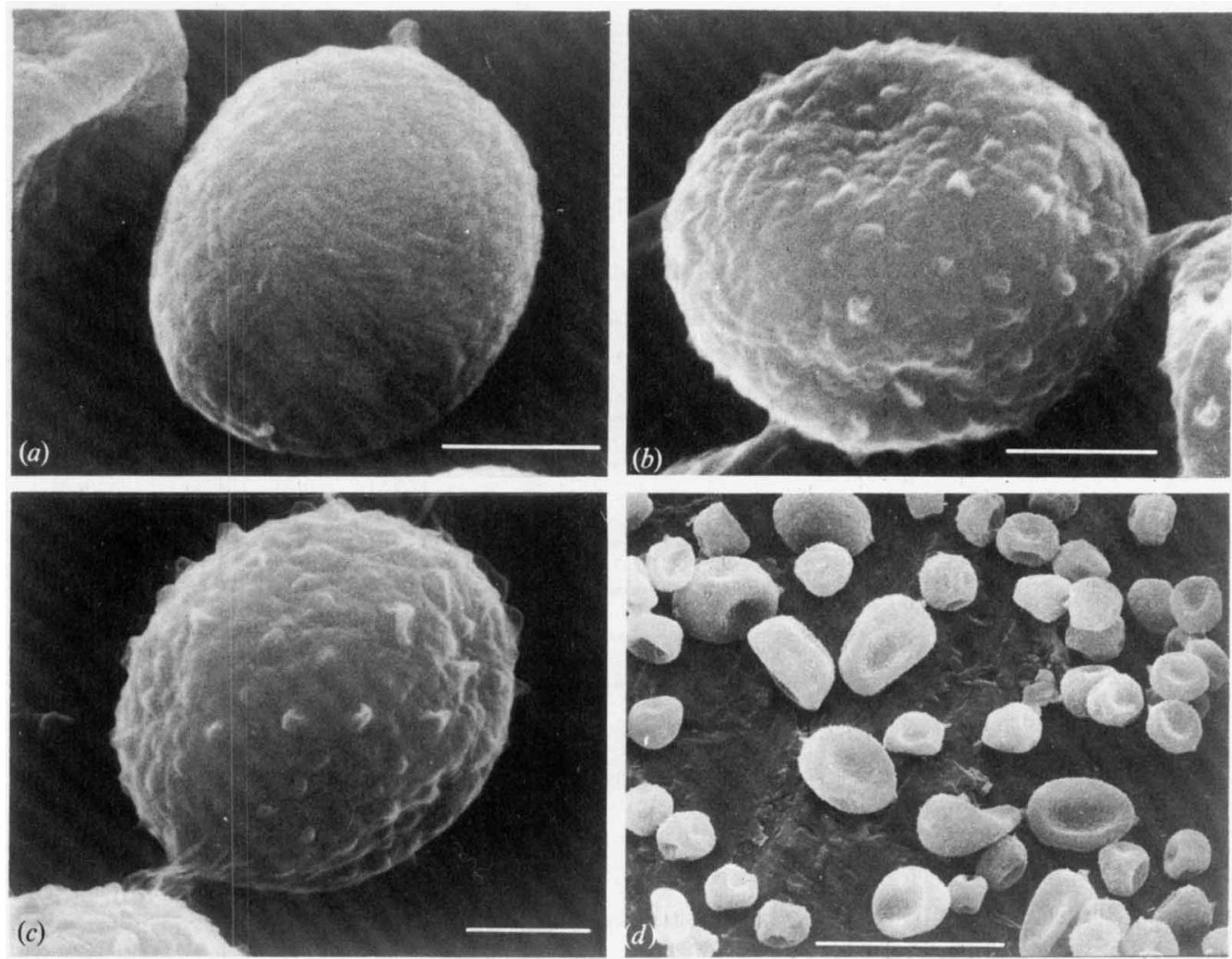

Fig. 2. Electron micrographs of conidia from single-conidium lines. (a) Conidia of type $1,(b)$ conidia of type $2,(c)$ conidia of type $3,(d)$ varying conidial size in line 1 . Bars represent $1 \mu \mathrm{m}$ in $(a)-(c), 10 \mu \mathrm{m}$ in $(d)$. This figure is Crown Copyright (C) 1987. 


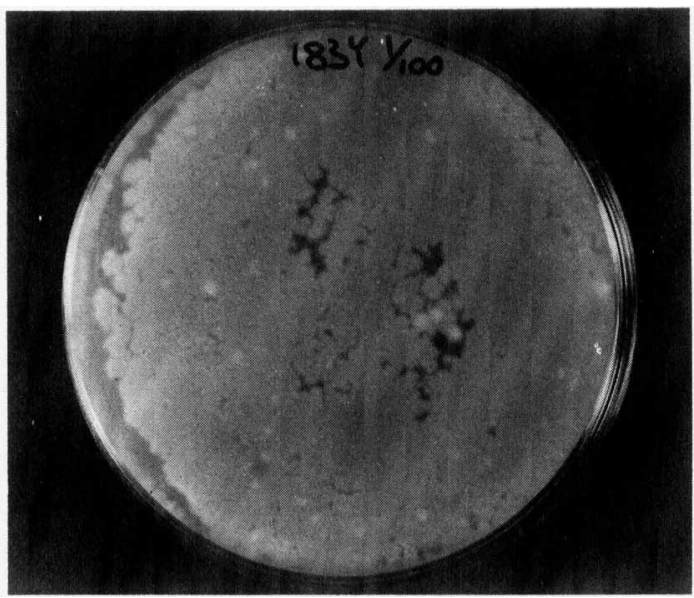

Fig. 3. Occurrence of pigment-deficient colonies similar to line $\mathrm{E}$ in a population derived from yellowpigmented line $\mathrm{A}$.

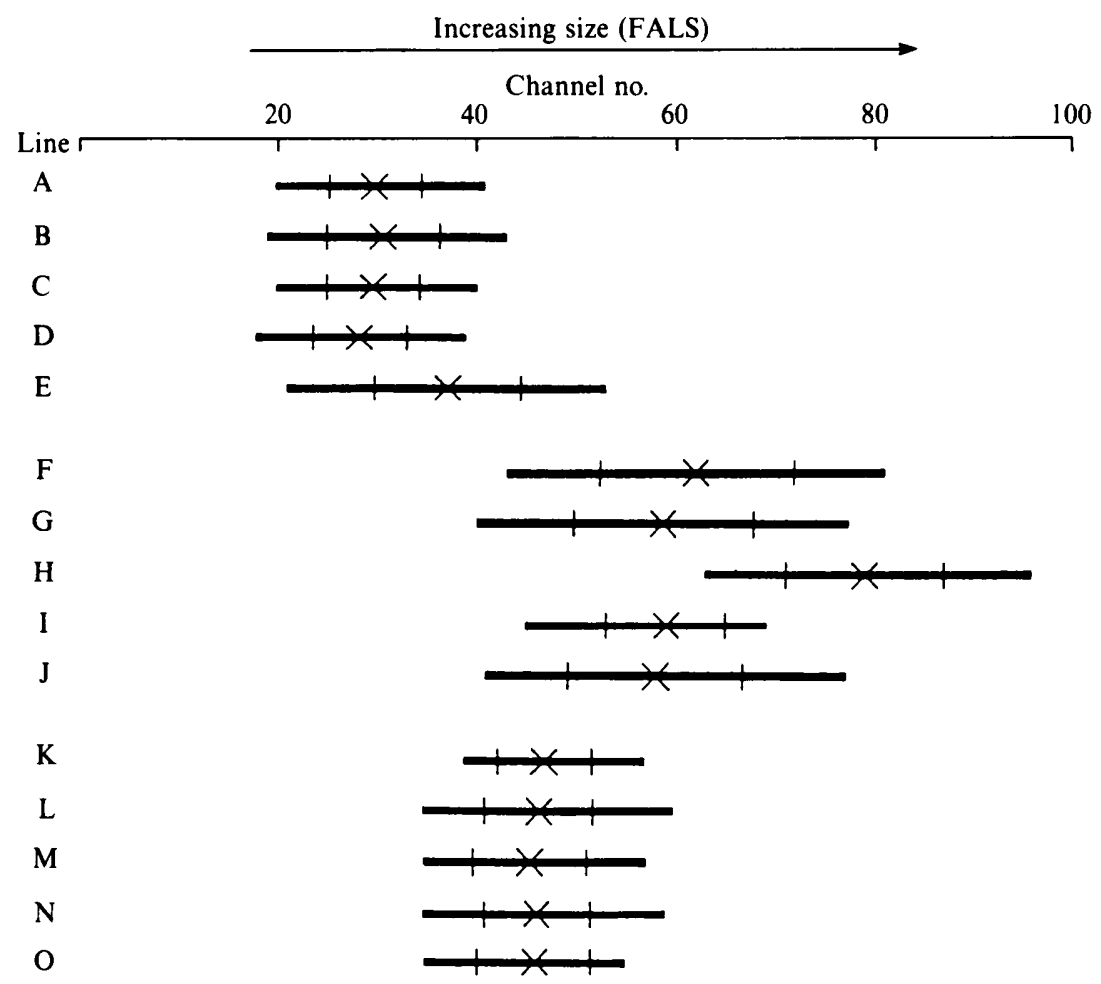

Fig. 4. Flow cytometer FALS measurements showing size variation in single-conidium lines. The horizontal bars indicate ranges; means are shown by crosses, and standard deviations by the vertical lines on either side of each cross.

single-conidium lines that showed wide variation in colony size (respective colony diameters 8 , $8,13,21,24$ and $28 \mathrm{~mm}$ after $21 \mathrm{~d}$ ).

Flow cytometric comparison of conidial sizes gave results broadly in agreement with the SEM studies, with the exception of lines E, M and N. FALS showed that the mean sizes within the different single-conidium lines varied approximately with the parent culture, with lines A-D 

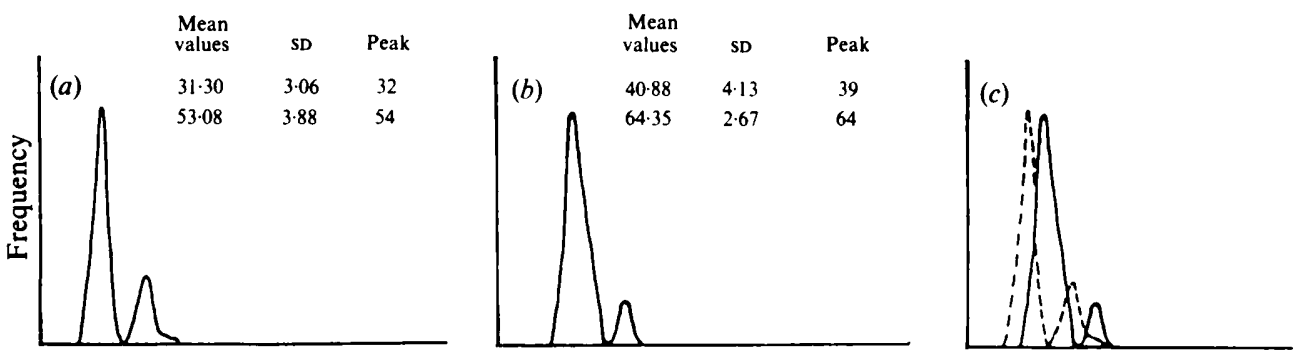

Log integral green fluorescence

Fig. 5. Examples of DNA profiles seen by measuring LIGFL. (a) Line B from $P$. granulatum, (b) line K from $P$. crustosum, $(c)$ superimposition of traces from lines $\mathrm{B}$ and $\mathrm{K}$ on the same scale.

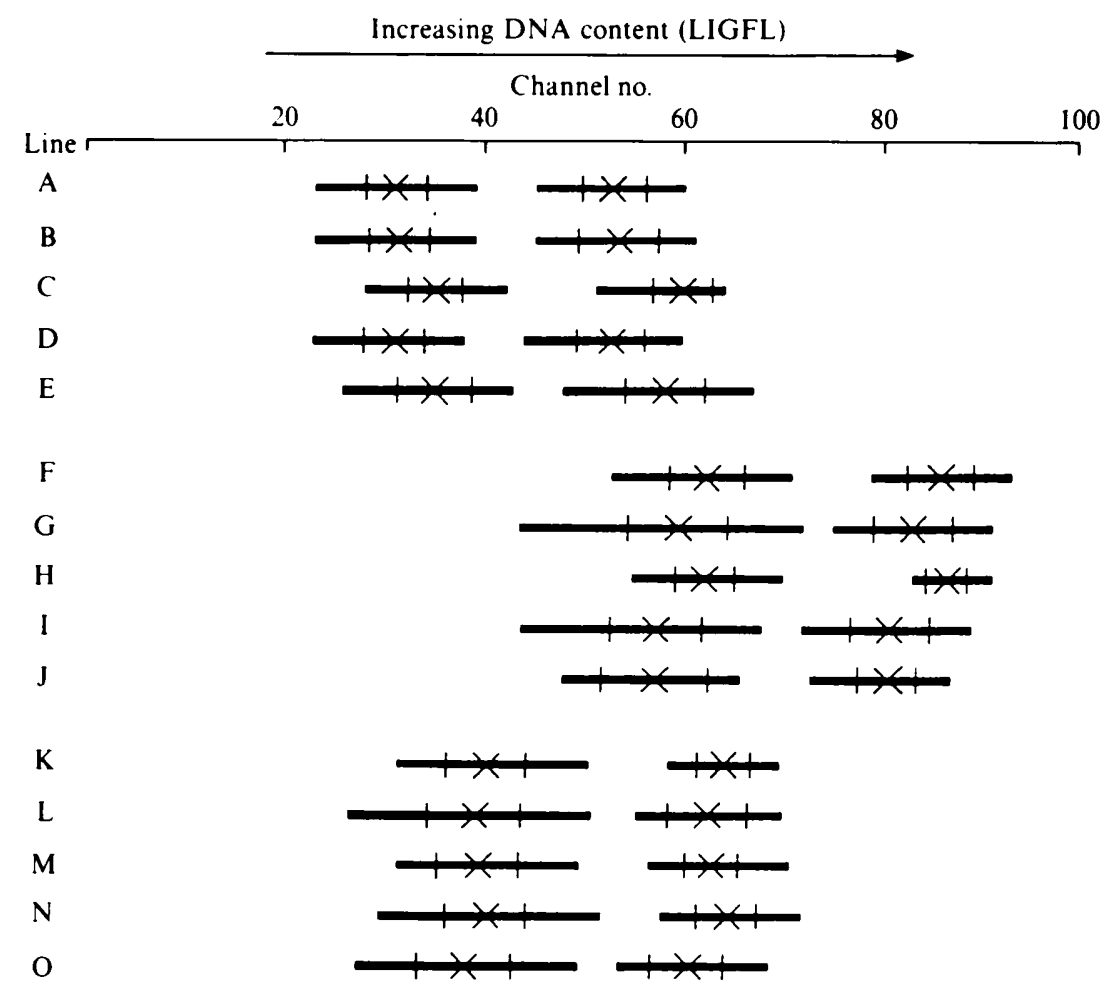

Fig. 6. Flow cytometer LIGFL mesurements showing variation in DNA content in single-conidium lines. Symbols as for Fig. 4.

from $P$. granulatum (SEM type 1) showing the smallest conidia and lines from P. martensii (SEM type 3) showing the largest. However, the overall size range for each line varied and there was some overlap between different lines (Fig. 4).

Measurements of conidial DNA content showed two peaks, the first containing the majority of the population (55-88\%), and the second appearing at approximately double the DNA content of the first (Fig. 5). The DNA contents corresponded with the conidial sizes: the least DNA was seen in the predominantly small conidium populations of $P$. granulatum, the intermediate-sized conidium populations of $P$. crustosum contained slightly more DNA, and the predominantly large conidium populations of $P$. martensii contained twice as much DNA as $P$. crustosum (see Fig. 6). This correspondence was, however, again only approximate and there 
was some variation between lines of the same culture. Lines $C$ and $E$ from P. granulatum showed higher DNA contents for both peaks than the other lines of this culture. These increases were less than the combined standard deviations for the peaks, but as they also corresponded with an upward shift in the range and were reproducible, they are probably significant.

\section{DISCUSSION}

In any study on strain characterization there will be at least two types of variations, those arising from the reproducibility of the test methods and those arising from variation within the population. In this investigation, variations in test methods were studied by duplicating one line, and treating the two lines separately. This showed that there was variation associated with some test methods. Conidial colours, production of straw-coloured exudate, and the presence of swollen supporting hyphae all showed significant differences between the duplicated lines. This type of difference may be attributable to subjective recording. In this study variation in test methods was minimized by doing all tests at least in duplicate at the same time and using media and reagents from the same batch.

The results show that significant differences can occur in phenotypic characterization due to variation within the population, and that the extent of this variation may differ widely between different isolates. This type of variation has been widely reported in fungi (e.g. Scharen \& Krupinsky, 1970) and it was suggested by Bridge et al. (1986 b) that in one strain of $P$. viridicatum this may be due to some form of chromosome duplication, such as aneuploidy. In this study the five single-spore lines of $P$. granulatum showed the greatest variation, clustering as two pairs and one loose isolate. Two of these five lines ( $C$ and $E$ ) had higher conidial DNA contents than the other three, and one of these (E) is the loosely linked isolate. This loosely linked isolate also showed larger conidia than the other lines from P. granulatum. There are a number of similarities between this case and that of $P$. viridicatum. In both cases the obvious morphological differences were colony size and pigment production, in both cases the larger unpigmented colony had more echinulate conidia, and in both cases the two lines were interconvertible at low frequencies. The upward shift in DNA content is seen for both of the peaks, indicating that it is associated with replicating nuclear DNA; it is therefore unlikely to involve mitochondrial DNA. However, the stability of the lines appears to be greater than that normally expected for aneuploidy, a possible explanation being a transposition following partial chromosome duplication (O'Donnell et al., 1985), and this could also account for the increase in conidial size in line E. One significant difference in the isolates used for this study, however, is the age of the cultures. The isolate of $P$. viridicatum (Bridge et al., 1986 b, c) used had been maintained in a culture collection for at least 30 years. In this study the original isolate of $P$. granulatum was received in 1985 and has to our knowledge only been subcultured five or six times at most.

The large conidia of the lines of $P$. martensii contained approximately twice as much DNA as those of the other isolates. This appears to be a considerable difference for isolates as apparently closely related as fasciculate penicillia. One possible explanation is that these isolates are relatively stable diploids, and this would agree with the general theory that chromosomal duplications are involved in phenotypic variation. Naturally occurring diploid isolates of some fungi, such as Aspergillus, have been reported, but the relative occurrence and importance of this feature is unclear (Upshall, 1981). Maintenance records at CMI report that this strain has a tendency to produce sectors in culture, but none were seen in this study.

The apparent discrepancies between the SEM and flow-cytometer results for the conidial sizes may be due to the relative sample sizes. The SEM examinations are based on approximately 50 conidia, while the flow-cytometer observations are from 10000 conidia, and so may be more representative. This does however highlight the problem of basing descriptions on a relatively small sample of a large population.

Characters found to vary in this study are, in some cases, the final expression of relatively large pathways. For example Bu'Lock (1980) has estimated that approximately 100 genes may be involved in the production of a single secondary metabolite. These pathways contain a number of control and regulation genes and so a relatively small alteration of the genome could 
significantly alter the secondary metabolite production. Interestingly, the secondary metabolites which showed variation between single-conidium lines (Table 1) are derived via different metabolic routes. Brefeldin $\mathrm{A}$ is a cyclopentane derived from oxidative cyclizations of unsaturated fatty acids, 11-deacetoxywortmannin is a terpenoid derived by metabolism of sterol, roquefortine $B$ is an ergot alkaloid derived from a single amino acid, whereas roquefortine $C$ is derived from mevalonate, tryptophan and histidine. The apparent variation in secondary metabolite formation seen in this study may be due to the levels of metabolite produced, lower levels being below the sensitivity of the TLC system. Wide variation in metabolite levels has been reported in single-conidium isolates of Aspergillus (Schroeder, 1969). Equally, a similar situation may be present in many of the other varying characters, such as enzyme or pigment production.

The object of this study was to provide information on characters that may vary within an isolate, as part of an integrated approach to Penicillium systematics (Bridge et al., 1986a). The second isolation of single conidia from $P$. granulatum suggested however that variation may be greater than reported here, as the widespread differences in colony diameter seen in the second isolation were unlike those seen in the first isolation.

The taxonomic significance of the variation reported here is considerable. Currently, Penicillium taxonomy depends primarily on morphological features such as colony size, colony and conidial colour, and conidial head structure (Samson et al., 1976; Pitt, 1980). Further subdivision on the basis of some physiological characters and secondary metabolite production has also been proposed (Ciegler et al., 1973; Frisvad, 1981). These are all now seen to be characters that may vary significantly within an isolate, and so may not necessarily be sound criteria for species delineation if treated in isolation. A further taxonomic consideration must be whether stock reference strains give an accurate representation of the wild-type populations. Variation, very similar to that described here, has been seen in wild-type isolates received at CMI. If significant variation occurs within the wild-type population (as the results for $P$. granulatum suggest), then selection may lead to the establishment of populations differing from earlier reference cultures. The appearance of spontaneous mutants in yeast populations has caused some problems with accurate speciation (Stahl \& Esser, 1979) and it is apparent that similar considerations must be made for filamentous fungi.

This study was carried out as part of SERC contract SO/17/84 'Systematics of microfungi of industrial and biotechnological importance'. We are grateful to Professor D. L. Hawksworth for his advice and encouragement in this work and for critical reading of the manuscript.

\section{REFERENCES}

BRIDGE, P. D. (1985). An evaluation of some physiological and biochemical methods as an aid to the characterization of species of Penicillium subsection Fasciculata. Journal of General Microbiology 131, 1887-1895.

BRIDGE, P. D. \& MAY, J. W. (1984). A numerical classification of fission yeasts of the genus Schizosaccharomyces Lindner. Journal of General Microbiology 130, 1921-1932.

Bridge, P. D., Hawksworth, D. L., Kozakiewicz, Z., Onions, A. H. S., Paterson, R. R. M. \& Sackin, M. J. (1986a). An integrated approach to Penicillium systematics. In Advances in Penicillium and Aspergillus Systematics, pp. 281-309. Edited by R. A. Samson \& J. I. Pitt. New York: Plenum.

Bridge, P. D., Hudson, L., Hawksworth, D. L. \& BRIDGE, D. A. (1986b). Variation in nuclear DNA content in an ex-type isolate of Penicillium measured by continuous flow microfluorimetry. FEMS Microbiology Letters 37, 241-244.

Bridge, P. D., Hawksworth, D. L., Kozakiewicz, Z., Onions, A. H. S. \& Paterson, R. R. M. (1986c).
Morphological and biochemical variation in single isolates of Penicillium. Transactions of the British Mycological Society 87, 389-396.

Bu'Lock, J. D. (1980). Mycotoxins as secondary metabolites. In The Biosynthesis of Mycotoxins: a Study in Secondary Metabolism, pp. 1-16. Edited by P. S. Steyn. New York: Academic Press.

Ciegler, A., Fennell, D. I., Sansing, G. A., Detroy, R. W. \& BenNetT, G. A. (1973). Mycotoxinproducing strains of Penicillium viridicatum: classification into subgroups. Applied Microbiology 26, 271278.

Frisvad, J. (1981). Physiological criteria and mycotoxin production as aids in identification of common asymmetric penicillia. Applied and Environmental Microbiology 41, 568-579.

HoOKer, A. L. (1957). Cultural variability in Septoria avenae through successive single-macrospore transfers. Phytopathology 47, 460-468.

KAFER, E. \& UPSHALl, A. (1973). The phenotypes of the eight disomics and trisomics of Aspergillus nidulans. Journal of Heredity 64, 35-38. 
Minter, D. W., Hawksworth, D. L., Onions, A. H. S. \& KoZAKIEWICZ, Z. (1986). Descriptive terminology of the conidiogenous structures in Aspergillus and Penicillium. In Advances in Penicillium and Aspergillus Systematics, pp. 71-88. Edited by R. A. Samson \& J. I. Pitt. New York: Plenum.

O'Donnell, C. H., Upshall, A. \& Macdonald, K. D. (1985). Gene dosage effects and antibiotic synthesis in fungi. In Gene Manipulations in Fungi, pp. 293309. Edited by J. W. Bennett \& L. L. Lasure. London: Academic Press.

Onions, A. H. S., BRIDge, P. D. \& PATERson, R. R. M. (1984). Problems and prospects for the taxonomy of Penicillium. Microbiological Sciences 1, 185-189.

PATERSon, R. R. M. (1986). Standardized one and two dimensional thin layer chromatographic methods for the identification of secondary metabolites in Penicillium and other fungi. Journal of Chromatography 368, 249-264.

Pathax, S. G. \& Elander, R. P. (1971). Biochemical properties of haploid and diploid strains of Penicillium chrysogenum. Applied Microbiology 22, 366-371.

PITT, J. I. (1980). The Genus Penicillium and its Teleomorphic States Eupenicillium and Talaromyces. London: Academic Press.

Samson, R. A. \& Gams, W. (1984). The taxonomic situation in the hyphomycete genera Penicillium, Aspergillus and Fusarium. Antonie van Leeuwenhoek 50, 815-824.
Samson, R. A., Stolk, A. C. \& Hadlock, R. (1976). Revision of the subsection Fasciculata of Penicillium and some allied species. Studies in Mycology, Baarn 11, $1-47$.

SCHAREN, A. L. \& Krupinsky, J. M. (1970). Cultural and inoculation studies of Septoria nodorum, cause of glume blotch of wheat. Phytopathology 60, 14801485.

SCHROEDER, H. W. (1969). Factors influencing the development of aflatoxins in some field crops. Journal of Stored Product Research 5, 187-192.

Smith, D. \& ONIONS, A. H. S. (1983). The Preservation and Maintenance of Living Fungi. Kew: Commonwealth Mycological Institute.

SNeath, P. H. A. \& Sokal, R. R. (1973). Numerical Taxonomy. The Principles and Practice of Numerical Classification. San Francisco: Freeman.

STAHL, U. \& ESSER, K. (1979). Inconsistency in the species concept for yeasts due to mutations during vegetative growth. European Journal of Applied Microbiology and Biotechnology 8, 271-278.

TOLMSOFF, W. J. (1983). Heteroploidy as a mechanism of variability among fungi. Annual Review of Phytopathology 21, 317-340.

UPSHALl, A. (1981). Naturally occurring diploid isolates of Aspergillus nidulans. Journal of General Microbiology 122, 7-10. 